\title{
Editorial
}

\section{International workshop on the biology of lactation in farm animals}

\author{
H. Sauerwein ${ }^{1} \odot$ and R. M. Bruckmaier ${ }^{2 \dagger} \oplus$ \\ ${ }^{1}$ Institute of Animal Science, Physiology and Hygiene Unit, University of Bonn, 53115 Bonn, Germany; ${ }^{2}$ Veterinary Physiology, Vetsuisse Faculty, University of Bern, \\ CH-3012 Bern, Switzerland
}

This special issue focusing on 'Biology of Lactation in Farm Animals' (BOLFA) presents invited review papers that are related to presentations during the BOLFA workshop in Dubrovnik, Croatia, in 2018. The workshop is traditionally (since 1992) held every 2 years, mostly in conjunction with the annual meetings of either European Federation of Animal Science (EAAP) or American Association of Animal Science (ASAS) or Joint Annual meeting of ASAS and ADSA (JAM).

Many aspects of lactation in farm animals differ considerably from conditions in wild animals or humans because domestication and breeding have increased milk yield in dairy animals, or the number of offspring in polytocous species, that is, in swine. The biology of lactation in farm animals therefore deals mainly with aspects of mammary gland function and related topics in livestock animals that were substantially changed by genetic selection for production traits important for the needs of both producers and consumers.

In the present issue, the most important research topics in mammary gland biology in farm animals are critically addressed. The review papers deal with a broad range of themes ranging from mammary development, over regulation and manipulation of milk secretion at different stages of lactation, alternatives to the 305-day standard lactation in dairy cows, old and new indicators in milk for health status and metabolic load, towards new developments in milking routines.

\section{Mammary development}

The basics of mammary development during foetal and postnatal growth with the interrelationship of hormonal cues at puberty and gestation were considered as largely known in cattle. With recent findings contrasting the notion of postnatal mammary growth being largely isometric and showing that the allometric growth of the mammary gland also seems to depend on nutritional cues before weaning, new aspects not only for basic science but also for developing strategies

\footnotetext{
${ }^{\dagger}$ E-mail: rupert.bruckmaier@vetsuisse.unibe.ch
}

for a targeted support of mammary development emerged. The most recent findings were summarised and provide an updated overview on mammary development in dairy calves (Geiger, 2019).

For a polytocous species, the pig, a comprehensive outline about mammary gland development is provided, starting with the very early embryonic stages when first primordia of the mammary glands appear over the following morphogenetic steps until birth and thereafter. In swine, postnatal mammary growth is isometric but turns allometric towards puberty. The importance of the hormonal cues related to puberty and later on in pregnancy is presented providing an in-depth insight in the respective functions of individual hormones, for example prolactin, until early lactation, when the secretory stage is reached (Hurley, 2019).

The importance of suckling in pigs is also reviewed starting from the cyclic changes of mammary development and involution during lactation and at weaning, towards the effects of teat use and duration of teat use for mammary development in the following lactation, and addressing also the importance of previous teat use and teat preference using intriguing experimental models (Farmer, 2019).

\section{Regulation and manipulation of milk secretion at different stages of lactation}

Most mammalian farm animal species are ungulates, and their newborn offspring depends on high-quality colostrum to ensure survival. New knowledge of colostrum formation is discussed by Quesnel and Farmer (2019). During lactation, the function of the mammary gland in dairy ruminants is affected by several exogenous factors such as milking frequency and feed supply, and endogenous factors, for example, stage of lactation, or genetics. The cellular mechanisms mediating the reaction towards such factors comprise secretory activity, epigenetic marks and changes in cell numbers are reviewed contrasting also reversible versus irreversible alterations of milk production (Boutinaud et al., 2019). 
The manipulation of milk secretion goes new directions. Instead of pushing the mammary towards higher production by manipulating various endocrine systems, new approaches aim at a transient reduction of production in phases when the genetic drive for milk yield might be compromising animal welfare and health. The major stages of interest are the very first days of lactation to facilitate the adaptation to lactation after the dry period and also at dry off if cows produce still very high amounts of milk, and it is a special challenge to achieve a cessation of milk secretion. New results revising the role of prolactin for lactation in ruminants are reviewed focusing on the importance of prolactin inhibitors for transiently reducing milk yield (Lacasse et al., 2019).

\section{Alternatives to the 305-day standard lactation}

The increase of milk yields achieved by genetic and, more recently, genomic selection, has also triggered considerable concerns about animal health in dairy cows. Besides the problems related to the negative energy balance in early lactation, high milk yields towards drying-off might impose compromised welfare and health. With this background, two new concepts emerged during the past decade for circumventing these problems: one takes variations in dry period length, ranging from complete omission of a dry period to differing lengths of the dry period, into consideration. The long-term consequences for metabolism and welfare are presented, and feeding strategies for dealing with the increased energy balance during the entire lactation period counteracting overconditioning are outlined. The individual reactions observed necessitate individualised dry periods depending on cows' characteristics. Based on the literature available, such customised dry period lengths hold promise to reduce the metabolic stress and to improve health status and may also be beneficial for fertility (Kok et al., 2019).

A second way, aiming to extend the duration of the lactation cycle, is based on postponing the time for insemination for inducing a new pregnancy well beyond the 'normal' time in order to achieve longer lactation periods. A comprehensive overview of the advantages and disadvantages of extended lactation periods is provided addressing also management and efficiency issues (Sehested et al., 2019). Using model calculations, optimised lactation lengths depending on lactation number are presented. An individual selection of which cows are most suitable for extended lactation may further help implementing extended lactation strategies in praxis.

\section{Old and new indicators in milk for health status and metabolic load}

Milk samples are classically used for assessing udder health and diagnosing mastitis. Besides, the use of milk as sample matrix for also evaluating metabolic health gained increasing interest. Due to the ease of non-invasive sample collection, the possibility of implementing such analyses into routine control measures using mid-infrared as high throughput analyses is increasingly exploited. Focusing on ketosis as an important metabolic disease, the use of indicators, mainly B-hydroxybutyrate in milk, is reviewed trading off the benefits and limitations of applying such measurements (Gross and Bruckmaier, 2019).

With regard to mastitis, not only the advancement in proteomic but also metabolomics approaches in milk are compiled in a further review focusing on Streptococcus uberis infection as an experimental model. The identification of proteins and metabolites being up- or down-regulated in infected versus healthy animals is elucidating the defence reactions and extends our knowledge about factors that are relevant in this context (Eckersall, 2019).

\section{New developments in milking routines}

Two review papers in the issue deal with milk removal in dairy cows (Odorcic et al., 2019), and in small dairy ruminants (Dzidic et al., 2019). A fast but gentle removal of milk by machine milking is aimed at in science and is increasingly implemented in milking routines on practical farm. For this, fast milking is combined with an early detachment of the teat cups in both conventional and robotic systems. This is a different approach compared to the past (partially still used) when the philosophy was a time-consuming complete emptying of the udder by the milking machine, often even followed by hand-stripping. This has been shown to cause a considerable mechanical load on the teat tissue, and leaving small amounts of milk in the udder seems to be advantageous compared to teat tissue damage and hyperkeratosis (Odorcic et al., 2019).

The Biology of Lactation in Farm Animals in all its complexity remains a central theme in animal and dairy science. The current collection of reviews provides the state-of-the-art knowledge as well as emerging concepts and is thus an indispensable update for both research and teaching.

\section{Acknowledgements}

Financial assistance to the workshop on the 'Biology of Lactation in Farm Animals' by the sponsors listed alphabetically below is gratefully acknowledged:

American Society of Animal Science (ASAS), Animal Consortium, BASF, DeLaval, Diamond V, European Federation of Animal Science (EAAP), Förster Technik, GEA and Zinpro.

Helga Sauerwein, 0000-0002-6905-4053

Rupert M. Bruckmaier 0000-0002-9374-5890

\section{Declaration of interest}

None.

\section{Ethics statement}

None. 


\section{Software and data repository resources}

This is a collection of review papers where relevant statements about deposition of data or models in an official repository are referenced in the individual review articles and/or the articles listed as references therein.

\section{References}

Boutinaud $M$, Hervé $L$, Quesnel $H$, Lollivier V, Finot $L$, Dessauge $F$, Chanat $E_{\text {, }}$ Lacasse P, Charton C and Guinard-Flament J 2019. Review: The cellular mechanisms underlying mammary tissue plasticity during lactation in ruminants. Animal, 13 s52-s64.

Dzidic A, Rovai M, Poulet JL, Leclerc M and Marnet PG 2019. Review: Milking routines and cluster detachment levels in small ruminants. Animal, 13 s86-s93.

Eckersall PD 2019. Review: Proteomic approaches to control lactational parameters in dairy cows. Animal, 13 s82-s85.

Farmer C 2019. Review: Mammary development in lactating sows: the importance of suckling. Animal, 13 s20-s25.
Geiger AJ 2019. Review: The pre-pubertal bovine mammary gland: unlocking the potential of the future herd. Animal, 13 s4-s10.

Gross JJ and Bruckmaier RM 2019. Review: Metabolic challenges in lactating dairy cows and their assessment via established and novel indicators in milk. Animal, 13 s75-s81.

Hurley WL 2019. Review: Mammary gland development in swine: embryo to early lactation. Animal, 13 s11-s19.

Kok A, Chen J, Kemp B and van Knegsel ATM 2019. Review: Dry period length in dairy cows and consequences for metabolism and welfare and customised management strategies. Animal, $13 \mathrm{~s} 42-\mathrm{s} 51$.

Lacasse $P$, Zhao X, Vanacker N and Boutinaud M 2019. Review: Inhibition of prolactin as a management tool in dairy husbandry. Animal 13 s35-s41.

Odorcic M, Rasmussen MD, Paulrud CO and Bruckmaier RM 2019. Review: Milking machine settings, teat condition and milking efficiency in dairy cows. Animal, 13 s94-s99.

Quesnel H and Farmer C 2019. Review: Nutritional and endocrine control of colostrogenesis in swine. Animal, 13 s26-s34.

Sehested J, Gaillard C, Lehmann JO, Maciel GM, Vestergaard M, Weisbjerg MR, Mogensen L, Larsen LB, Poulsen NA and Kristensen T 2019. Review: Extended lactation in dairy cattle. Animal, 13 s65-s74. 